\title{
THE UNUSUAL SUPER-LUMINOUS SUPERNOVAE SN 2011KL AND ASASSN-15LH
}

\author{
Melina C. Bersten ${ }^{1,2,3}$, Omar G. Benvenuto ${ }^{1,2,4}$, Mariana Orellana ${ }^{5,6}$, and Ken'ichi Nomoto ${ }^{3,7}$ \\ ${ }^{1}$ Facultad de Ciencias Astronómicas y Geofísicas, Universidad Nacional de La Plata, Paseo del Bosque S/N, \\ B1900FWA La Plata, Argentina; mbersten@ fcaglp.unlp.edu.ar \\ ${ }^{2}$ Instituto de Astrofísica de La Plata (IALP), CONICET, Argentina \\ ${ }^{3}$ Kavli Institute for the Physics and Mathematics of the Universe, The University of Tokyo, \\ 5-1-5 Kashiwanoha, Kashiwa, Chiba 277-8583, Japan \\ ${ }^{4}$ Member of the Carrera del Investigador Científico de la Comisión de Investigaciones Científicas de la Provincia de Buenos Aires (CIC), Argentina \\ ${ }_{6}^{5}$ Sede Andina, Universidad Nacional de Río Negro, Mitre 630 (8400) Bariloche, Argentina \\ ${ }^{6}$ Member of the Carrera del Investigador Científico y Tecnológico del CONICET, Argentina \\ Received 2015 November 11; accepted 2015 December 27; published 2016 January 19
}

\begin{abstract}
Two recently discovered very luminous supernovae ( $\mathrm{SNe}$ ) present stimulating cases to explore the extents of the available theoretical models. SN $2011 \mathrm{kl}$ represents the first detection of a supernova explosion associated with an ultra-long duration gamma-ray burst. ASASSN-15lh was even claimed as the most luminous SN ever discovered, challenging the scenarios so far proposed for stellar explosions. Here we use our radiation hydrodynamics code in order to simulate magnetar-powered SNe. To avoid explicitly assuming neutron star properties, we adopt the magnetar luminosity and spin-down timescale as free parameters of the model. We find that the light curve (LC) of SN $2011 \mathrm{kl}$ is consistent with a magnetar power source, as previously proposed, but we note that some amount of ${ }^{56} \mathrm{Ni}\left(\gtrsim 0.08 M_{\odot}\right)$ is necessary to explain the low contrast between the LC peak and tail. For the case of ASASSN$15 \mathrm{lh}$, we find physically plausible magnetar parameters that reproduce the overall shape of the LC provided the progenitor mass is relatively large (an ejecta mass of $\approx 6 M_{\odot}$ ). The ejecta hydrodynamics of this event is dominated by the magnetar input, while the effect is more moderate for SN 2011kl. We conclude that a magnetar model may be used for the interpretation of these events and that the hydrodynamical modeling is necessary to derive the properties of powerful magnetars and their progenitors.
\end{abstract}

Key words: gamma-ray burst: individual (GRB 111209A) - stars: evolution - supernovae: general - supernovae: individual (SN 2011kl, ASASSN-15lh)

\section{INTRODUCTION}

Super-luminous supernovae (SLSNe) were discovered almost a decade ago (Quimby et al. 2007; Smith et al. 2007). They show a factor of 10-100 times brighter than normal corecollapse supernovae $(\mathrm{SNe})$, often well above a -22 absolute magnitude. It is believed that they are the explosion of massive stars, which are usually found in low luminosity, star forming dwarf galaxies (Neill et al. 2011; Lunnan et al. 2014; Leloudas et al. 2015). However, the physical origin of the extreme luminosity emitted remains speculative (see, e.g., GalYam 2012, for a review). The high luminosity of SLSNe makes them ideal resources to obtain information from the early universe and to explore the capability of using them as cosmological standard candles at a much further distance than normal SNe (Quimby et al. 2011; Inserra \& Smartt 2014).

In order to be radioactively powered, as normal SNe, SLSNe would require a nickel mass that is too large (except for pair instability SNe and hypernovae; see e.g., Moriya et al. 2010) thus competing alternatives have been proposed. One possible mechanism invoked to explain SLSNe is the energy injection by an accreting black hole that launches relativistic jets, or the fallback scenario (e.g., Dexter \& Kasen 2013 and references therein). The interaction of the SN ejecta with dense circumstellar material (CSM) is another proposed mechanism (but see Sorokina et al. 2015). Another popular explanation is that a magnetar is formed by the collapse of a massive star. The magnetar is a strongly magnetized, rapidly rotating neutron star (NS) that loses rotational energy via magnetic dipole radiation.

\footnotetext{
7 Hamamatsu Professor.
}

Although some progress in the area was recently reported (see, e.g., Timokhin \& Harding 2015), other important aspects of the scenario are still unclear.

In this work, we study two peculiar SLSNe of Type Ic (lacking hydrogen), SN 2011kl and ASASSN-15lh. SN 2011kl has been associated with the ultra-long-duration gamma-ray burst, GRB 111209A, at a redshift $z$ of 0.677 (Greiner et al. 2015). Its light curve (LC) was significantly overluminous compared to other GRB-associated SNe, suggesting for the first time a link between SN-GRB and SLSNe-ultra long GRB (ULGRB). The precise explosion time estimation makes this SN ideal for numerical modeling.

More recently, ASASSN-15lh at $z=0.2326$ was discovered by the All-Sky Automated Survey for SuperNovae (Dong et al. 2015). It showed a hydrogen-poor spectrum, and the maximum luminosity was $\sim 2.2 \times 10^{45} \mathrm{erg} \mathrm{s}^{-1}$, i.e., more luminous than any previously known SN. Contrary to SN $2011 \mathrm{kl}$, the explosion date of ASASSN-15lh is unknown, and data before the luminous peak were less reliable than later. The optical emission was continuously detected for more than 100 days after the explosion. The spectrum lacked the broad $\mathrm{H} \alpha$ emission feature that would have evidenced an interaction between the supernova and an H-rich circumstellar environment (Milisavljevic et al. 2015).

Magnetar models have been proposed for the two SLSNe of this work (Greiner et al. 2015; Metzger et al. 2015), even for the extremely luminous ASASSN-15lh. However, the analysis was based on simplistic assumptions that neglected the dynamic effects on the ejecta. Here we shall study the magnetar scenario using hydrodynamic calculations, which incorporate 


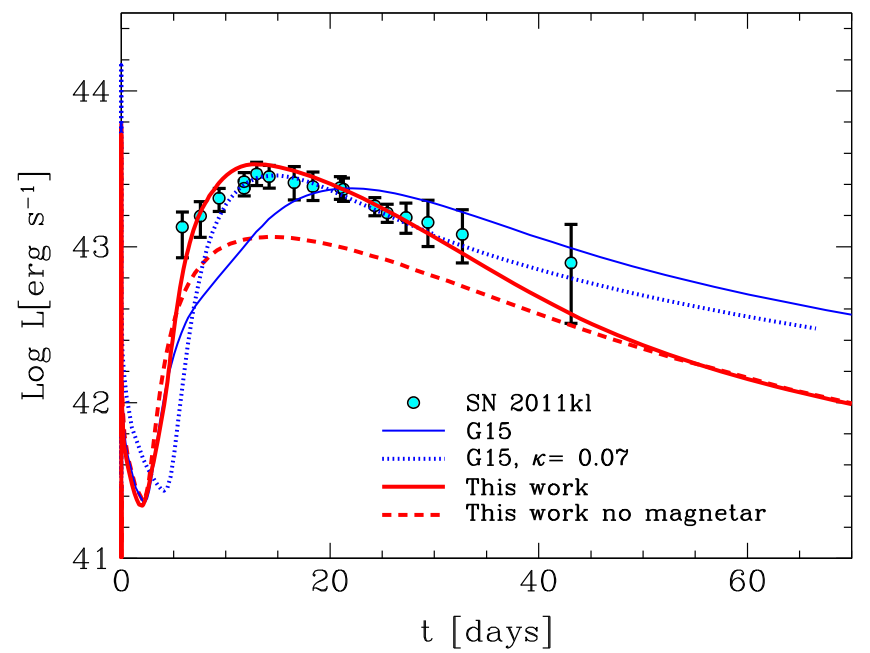

Figure 1. Bolometric LC of SN 2011kl compared with several LC models. The thick red solid line shows our preferred model with $P_{0}=3.5 \mathrm{~ms}$, and magnetic field $B=1.95 \times 10^{15} \mathrm{G}$ (see the text for more details). A model with $P_{0}=12.2 \mathrm{~ms}$ and $B=7.5 \times 10^{14} \mathrm{G}$, as suggested by $\mathrm{G} 15$, computed with our hydrodynamical modeling and our opacity prescription is shown by the thin blue line and the dotted line for $\kappa=0.07 \mathrm{~cm}^{2} \mathrm{~g}^{-1}$. A ${ }^{56} \mathrm{Ni}$ power model with a ${ }^{56} \mathrm{Ni}$ mass of $1 M_{\odot}$ is shown by the dashed line.

the dynamical effect and consider the limit imposed by the NS matter equation of state (EOS).

\section{MAGNETAR MODELS}

We include an extra source of energy due to a rapidly rotating and strongly magnetized NS (or "magnetar") in our one-dimensional LTE radiation hydrodynamic code (Bersten et al. 2011). A spherical young magnetar releases its rotational energy at a well-known rate described by the radiating magnetic dipole (e.g., Shapiro \& Teukolsky 1983). We assume that this energy is fully deposited and thermalized in the innermost layers of our pre-supernova model. This assumption as well as a large inclination $i=45^{\circ}$ is the same used in previous numerical works of magnetar-powered SN light curves (Kasen \& Bildsten 2010; Woosley 2010). Powerful enough magnetars may force the envelope to expand at velocities comparable to the speed of light (see below, particularly Figure 5). Therefore, we have modified our code to properly include relativistic velocities. A detailed description of our treatment of relativistic radiating hydrodynamics will be presented in a forthcoming paper.

Our hydrodynamical calculations simulate the explosion of an evolved star, followed consistently until the core-collapse condition. The pre-SN He star models of different masses used in this paper were calculated by Nomoto \& Hashimoto (1988). The explosion itself is simulated as usual by the injection of a certain energy (a few $\times 10^{51} \mathrm{erg}$ ) at the innermost layers of our pre-SN models. In addition to the explosion energy, the rotational energy lost by a newly born magnetar is included.

Once the NS momentum of inertia and radius $R$ are fixed, this energy source essentially depends on two parameters, the strength of the dipole magnetic field ${ }^{8}, B$, and the initial rotational period, $P_{0}$ (see, e.g., Equations (1) and (2) of Kasen $\&$ Bildsten 2010). It is equally possible to use the spin-down timescale $\left(t_{\mathrm{p}}\right)$ and magnetar energy loss rate $\left(L_{\mathrm{p}}\right)$ as the free

\footnotetext{
8 Considered fixed during the evolution.
}

parameters to be determined by fitting the observed LC. Therefore, the magnetar luminosity can be written as

$$
L=L_{\mathrm{p}}\left(1+\frac{t}{t_{\mathrm{p}}}\right)^{-2},
$$

with

$$
L_{\mathrm{p}}=\frac{4 \pi^{4} R^{6}}{3 c^{3}} \frac{B^{2}}{P^{4}}
$$

and

$$
t_{\mathrm{p}}=\frac{3 c^{3} I_{N S}}{\pi^{2} R^{6}} \frac{P^{2}}{B^{2}} .
$$

The values of $B$ and $P_{0}$ can be found later from $t_{\mathrm{p}}, L_{\mathrm{p}}$, assuming an NS mass. Then, the radius and momentum of inertia are found for a given EOS. In this way, one avoids the assumptions made beforehand regarding explicit values of the NSs properties. This method allows for the possibility to accommodate a variety of NS structures, which is relevant because of our poor knowledge of the EOS at these extreme conditions.

In the calculation, if the photosphere recedes deep enough so that part of the magnetar energy is directly deposited outside the photosphere, we add this energy to the bolometric luminosity. The same treatment is used for ${ }^{56} \mathrm{Ni}$ deposition (Swartz et al. 1991; Bersten et al. 2011). We implicitly assume that the magnetar (or the ${ }^{56} \mathrm{Ni}$ decay) produces hard photons than can be trapped even if the ejecta is optically thin to optical photons. During these epochs, usually after about 60 days (although this depends on the progenitor mass and energy), the emitted luminosity is almost the same as the magnetar luminosity.

\section{RESULTS}

\subsection{Models for $S N 2011 \mathrm{kl}$}

Greiner et al. (2015; hereafter G15), recently analyzed the LC and spectrum of SN $2011 \mathrm{kl}$. A low ejecta mass $\approx 2.4 M_{\odot}$ and high explosion energy $\approx 5.5 \times 10^{51} \mathrm{erg}$ were obtained from their analysis. The radiative output of SN $2011 \mathrm{kl}$ locates it in between the GRB-SNe and SLSNe. A large ${ }^{56} \mathrm{Ni}$ mass $\approx 1 M_{\odot}$ was found necessary in order to reproduce the high observed luminosity. This large amount of radioactive material seems to be neither compatible with the spectrum properties nor with the low-mass ejecta, as pointed out by G15. A magnetar source was then proposed by the authors to explain the LC.

We used the code describe in Section 2 to study SN 2011kl. One advantage of the hydrodynamics calculations is that the explosion time $\left(t_{\exp }\right)$ is fully determined from the assumed physical parameters. The GRB detection of SN $2011 \mathrm{kl}$ establishes a tight value of $t_{\exp },{ }^{9}$ thus it is an ideal target of study. Motivated by the values proposed by G15, we assumed a pre-SN He star of $4 M_{\odot}$ with a cut mass $\left(M_{\text {cut }}\right)$ of $1.5 M_{\odot}$ corresponding to an ejected mass $\left(M_{\mathrm{ej}}\right)$ of $2.5 M_{\odot}$ and an explosion energy of $5.5 \times 10^{51} \mathrm{erg}$. While the LC of SN $2011 \mathrm{kl}$ is unlikely to be powered only by ${ }^{56} \mathrm{Ni}$, some amount of radioactive material is usually expected to be produced during the explosion. We assume a ${ }^{56} \mathrm{Ni}$ mass of

\footnotetext{
9 Here we assume that the GRB precursor indicated the explosion itself. However, see Vietri \& Stella (1999) for other possible scenarios.
} 


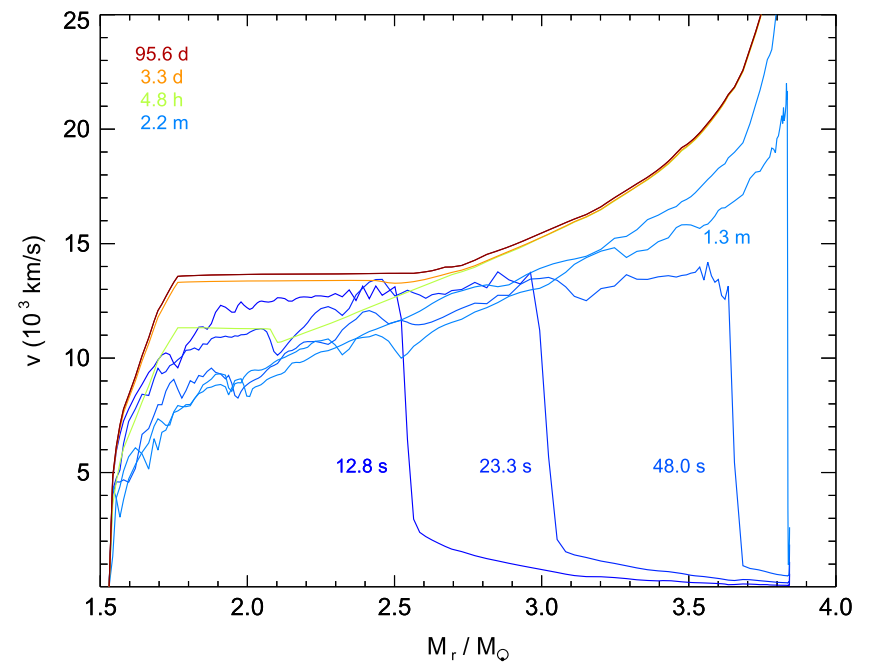

Figure 2. Velocity profile for the explosion that reproduces the LC of SN 2011kl. The interior dynamics is affected in comparison to a standard explosion without any magnetar. A day after explosion, the bulk of the stellar mass expands at $\approx 13 \times 10^{3} \mathrm{~km} \mathrm{~s}^{-1}$, or higher, whereas the outermost layers (not shown in the figure) reach velocities even higher (up to $0.21 \mathrm{c}$ ).

$0.2 M_{\odot}$ consistent with a radioactive yield of energetic SN (Nomoto et al. 2013).

We extensively explored the magnetar parameter space and found a reasonable agreement with the data for $P_{0}=3.5 \mathrm{~ms}$ and magnetic field $B=1.95 \times 10^{15} \mathrm{G}$. Figure 1 shows this model with a thick solid line. Our values lie out of the ranges given by $\mathrm{G} 15$ whose analysis gave $P_{0}=12.2 \pm 0.3 \mathrm{~ms}$ and $B=7.5 \pm 1.5 \times 10^{14} \mathrm{G}$. The thin line of Figure 1 shows the result of our hydrodynamical calculations assuming the same parameters as in G15. We found a poor match to the data as opposed to G15 (see their Figure 2). We note that we have used OPAL opacity tables with an opacity floor of $0.2 \mathrm{~cm}^{2} \mathrm{~g}^{-1}$ corresponding to the electron scattering opacity for hydrogen free material. This value is usually assumed as gray opacity in LC magnetar models in the literature (Kasen \& Bildsten 2010; Inserra et al. 2013). However, with a lower and constant value of $\kappa=0.07 \mathrm{~cm}^{2} \mathrm{~g}^{-1}$, presumably used in $\mathrm{G} 15$, we recovered a reasonable fit to the data for the magnetar parameters of G15 (see the dotted line in Figure 1).

In addition, we present our results for a model powered only by ${ }^{56} \mathrm{Ni}$. A model with $M\left({ }^{56} \mathrm{Ni}\right)=1 M_{\odot}$ is shown with a dashed line in Figure 1. Even with this large amount of ${ }^{56} \mathrm{Ni}$, we could not reach the high luminosity needed to explain SN 2011kl using our opacity prescription. While a nonstandard source of energy is necessary to explain the peak luminosity, some amount of ${ }^{56} \mathrm{Ni}\left(\gtrsim 0.08 M_{\odot}\right)$ is also needed to explain the tail luminosity. Magnetar models without nickel produce a larger contrast between peak and tail and thus a poor fit to the data.

Figure 2 shows the velocity profile at different times since the explosion for our preferred model (solid thick line of Figure 1). Initially, the shock wave propagates as in a standard explosion. Later the dynamic effect is noticeable, though not as extreme as in the case of ASASSN-15lh (see below). The extra heating source, caused by the magnetar, swells the inner zones and produces larger velocities and a flat profile that rise steeply only at the outermost layers. The high ejecta velocity is consistent with the analysis by G15 who inferred that a large value of $v_{\mathrm{ph}}$ would explain the rather featureless spectrum by

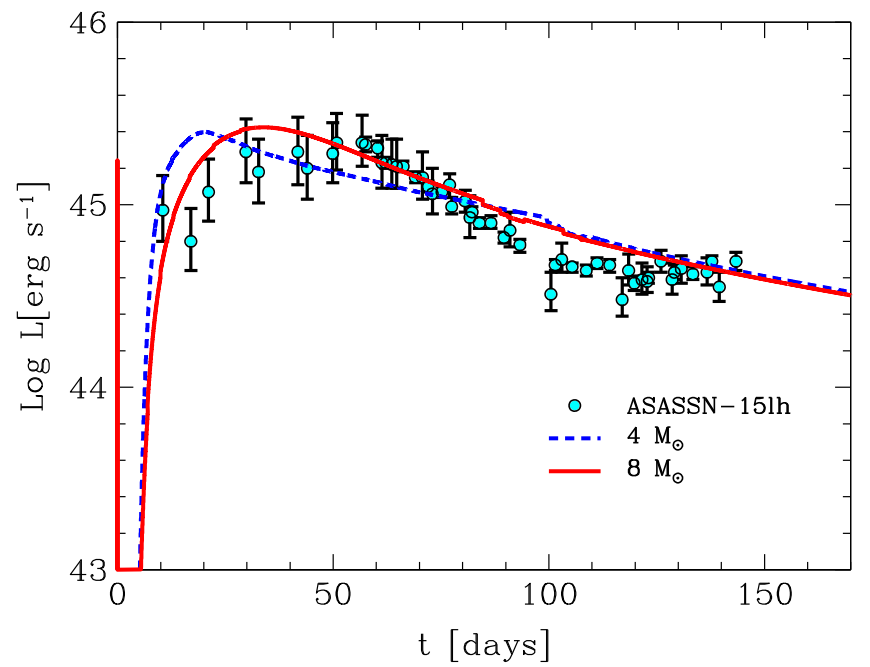

Figure 3. Observed bolometric LC of ASASSN-15lh (dots; Dong et al. 2015) compared with models of $4 M_{\odot}$ (dashed line) and $8 M_{\odot}$ (solid line) pre-SN mass for magnetar parameters of $L_{\mathrm{p}}=9 \times 10^{45}$ and $t_{\mathrm{p}}=40$ days and $t_{\exp }=$ JD 2457143.

Doppler line blending. We remark that here the opacity prescription is probably responsible for the differences found between our calculation and the analytic models presented by G15. The hydrodynamic effects of the magnetar on the luminosity are not as important as in the case of ASASSN-15lh, although, as shown in Figure 2, it is evident in the velocity evolution.

\subsection{Models for ASASSN-15lh}

Dong et al. (2015) reported the discovery of the brightest known SN, ASASSN-15lh with a peak luminosity of $\approx 2$ $\times 10^{45} \mathrm{erg} \mathrm{s}^{-1}$, almost two magnitudes brighter than the average observed SLSNe, and with a total radiated energy of $\approx 10^{52} \mathrm{erg}$. The spectra presented some similarities to SLSNeIc. Magnetar models seem to be the most plausible explanation for this class of SLSNe. However, Dong et al. (2015) emphasized the difficulty to explain this object in the magnetar context. The short initial period $\approx 1 \mathrm{~ms}$ required to match the LC properties could be in contradiction with the maximum rotational energy $\left(E_{\max }\right)$ available that, in turn, is limited by the emission of gravitational waves. However, Metzger et al. (2015) lately suggested that $E_{\max }$ could be up to one order of magnitude larger than previous estimations, depending on the NS properties, but avoiding the problem raised by Dong et al. (2015).

We calculated a set of magnetar models to analyze the LC of ASASSN-15lh. Here we use $L_{\mathrm{p}}$ and $t_{\mathrm{p}}$ as free parameters for the fitting (see Section 2). In this way, we avoid including explicitly the properties of the NS. Once the LC fit is admissible, we derived $B$ and $P_{0}$ as functions of the magnetar mass (see bellow). Figure 3 shows our results for a pre-SN He star of $4 M_{\odot}$ with $M_{\text {cut }}=1.5 M_{\odot}$ and $M_{\mathrm{ej}}=2.5 M_{\odot}$ (thin red line) and for a more massive model of $\mathrm{He}$ star $8 M_{\odot}$ with $M_{\text {cut }}=2 M_{\odot}$ and $M_{\text {ej }}=6 M_{\odot}$ (thick blue line). We assumed an initial explosion energy of $5.5 \times 10^{51} \mathrm{erg}$, though this has a minor effect on the results. Also, we assumed that the SN exploded a week before the discovery.

The magnetar parameters in this case are $L_{\mathrm{p}}=9 \times 10^{45} \mathrm{erg} \mathrm{s}^{-1}$ and $t_{\mathrm{p}}=40$ days. For the less massive 


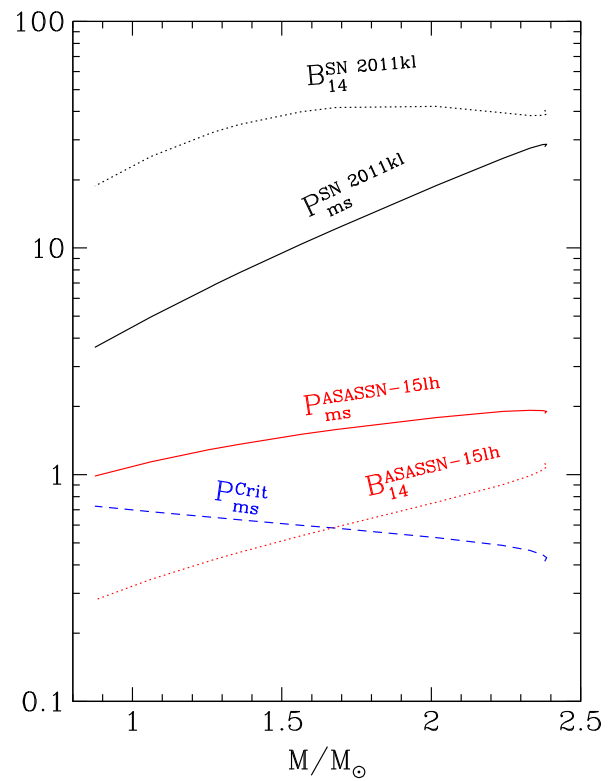

Figure 4. Magnetic fields (in units of $10^{14} \mathrm{G}$, represented with dotted lines) and initial spin periods (in milliseconds, denoted with solid lines) of the magnetar as a function of its mass. Black lines correspond to the parameters for SN 2011kl whereas red lines denote the case of ASSASN-15lh. For comparison, the critical spin period is given with a dashed line. Notice that for both cases the NS can rotate at the required rate.

model, we could not reproduce the overall LC shape. A more massive pre-SN model with $8 M_{\odot}$ or more is therefore needed to reproduce the temporal width of the LC. At $~ 100$ days, there is a slight change on the LC slope in coincidence with the moment that the photosphere reaches the inner regions where the magnetar energy is directly deposited. The observable effects of this fact should potentially modify the spectral energy distribution and deserve further investigation.

Figure 4 shows the values of $B$ and $P_{0}$ as functions of the NS mass derived from $L_{\mathrm{p}}$ and $t_{\mathrm{p}}$. For the structure of the magnetar, we have assumed the data presented in Table 12 of Arnett \& Bowers (1977). This corresponds to the structure of an NS assuming the nuclear matter EOS of Bowers et al. (1975), which gives a mass-radius relation similar to those currently favored by recent observations (see Figure 11(b) of Lattimer 2012). We have neglected rotational and magnetic effects on the NS structure. For comparison, we included the curve for the case of SN 2011kl, which represents the location of other possible solutions (i.e., the degeneracy of the parameters $B$ and $\left.P_{0}\right)$. Physically possible solutions correspond to rotation periods larger than the critical breakup value. For the complete NSs mass range, we found solutions that fulfill this condition. For the pre-SN model a specific value of $M_{\text {cut }}$ was assumed, but we have corroborated that changing this value in the range shown in Figure 4 produces a minor effect on the LC model.

We conclude that initial periods ranging between $\sim 1-2 \mathrm{~ms}$ and magnetic fields of $\sim(0.3-1) \times 10^{14} \mathrm{G}$, related by the curve of Figure 4, provide a reasonable fit to the LC. These magnetar values are in good agreement with those proposed by Metzger et al. (2015), though they assumed a lower ejecta mass of $3 M_{\odot}$. However, we could not reproduce the data with such a low-mass progenitor.

Here we should note that the uncertainty regarding $t_{\text {exp }}$ could modify the exact value of the parameters. In any case, the scope of this analysis is to show that the magnetar scenario is

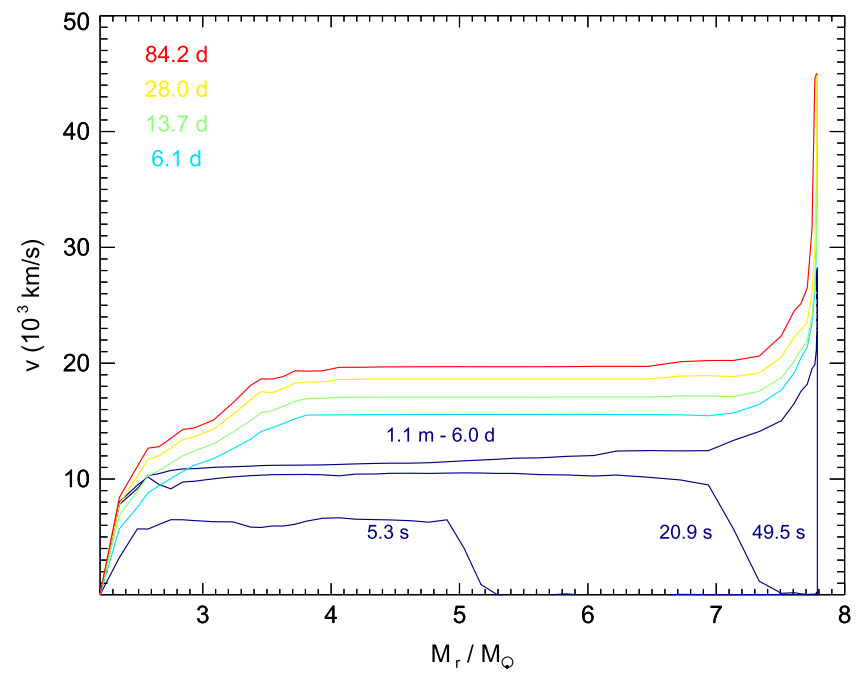

Figure 5. Velocity profile for ASASSN-15lh magnetar model with $L_{\mathrm{p}}=9 \times 10^{45}$ and $t_{\mathrm{p}}=40$ days. This extreme case inflates the external radial zones up to $0.15 \mathrm{c}$.

plausible for this object and not to provide definitive values of the physical parameters.

Figure 5 shows the velocity profiles for our preferred model of $8 M_{\odot}$. In this case, the magnetar is extremely powerful and the dynamical effect is more noticeable than for the case of SN 2011kl. The energy permanently supplied by the magnetar impulses all the envelope at high velocities and particularly the outermost layers. As a result, a few days after the explosion, most of the material moves at constant velocity, which increases with time due to the permanent injection of magnetar energy. This dynamic behavior should have a clear effect on the spectrum. Broad line features at 4100 and $4400 \AA$ were observed in the optical spectrum of ASASSN-15lh between 13 and 20 days after maximum implying very high velocities of $\approx 20.000 \mathrm{~km} \mathrm{~s}^{-1}$ (Metzger et al. 2015). This timing and velocity are fully consistent with the model shown in Figure 5. We note that the photosphere at this epoch is located in the flat part of the velocity profile.

\section{DISCUSSION AND CONCLUSIONS}

We were able to reproduce the LC of SN $2011 \mathrm{kl}$ and ASASSN-15lh in the context of magnetar-powered models with physically allowed parameters (the NS rotating below breakup point).

By adopting the magnetar luminosity and spin-down timescale as free parameters, we could separate the LC fit from any assumption on the NS structure. The usual parameters ( $B$ and $P_{0}$ ) could be recovered afterward by assuming the NS configuration for a given EOS. We have shown that this leads to a degeneracy between $B$ and $P_{0}$, which in turn depend on the properties of the nuclear matter EOS, which is poorly known.

For SN 2011kl, we found $L_{\mathrm{p}} \approx 1.2 \times 10^{45} \mathrm{erg} \mathrm{s}^{-1}$ and $t_{\mathrm{p}} \sim 15$ days. A family of values of $P_{0}$ and $B$ were derived that lie outside the ranges proposed by G15. We ascribe the differences to the opacity values adopted and not to the hydrodynamic effects. We note that in addition to the extra source of energy due to a magnetar, some non-negligible amount of ${ }^{56} \mathrm{Ni}\left(\gtrsim 0.08 \quad M_{\odot}\right)$ was also necessary in our calculation to produce the LC peak and tail. 
Regarding the extreme luminosity of ASASSN-15lh, the overall shape of the LC was reproduced for $L_{\mathrm{p}} \approx 9 \times 10^{45} \mathrm{erg} \mathrm{s}^{-1}$ and $t_{\mathrm{p}} \sim 40$ days. The resulting ranges of $B$ and $P_{0}$ were compatible with those proposed by Metzger et al. (2015). However, we note that our numerical models require a massive progenitor with $M_{\mathrm{ej}} \approx 6 M_{\odot}$, i.e., a factor of two larger than the value adopted by Metzger et al. (2015). Translating $M_{\mathrm{ej}}$ into a zero-age main-sequence mass, $M_{\mathrm{ZAMS}}$, of the progenitor involves several uncertainties. It is particularly important whether the He features can be hidden in the early spectra of SLSNe (Dessart et al. 2012; Hachinger et al. 2012). Assuming that this is the case, the pre-SN star could be a He star of $8 M_{\odot}$, which corresponds to $M_{\text {ZAMS }} \sim 25 M_{\odot}$ (see Tanaka et al. 2009 for a relation between pre-SN mass and $\left.M_{\text {ZAMS }}\right)$. On the other hand, if no He is present, then the pre-SN star could be a $\mathrm{C}+\mathrm{O}$ star of $8 M_{\odot}$, which corresponds to a $M_{\text {ZAMS }} \sim 30 M_{\odot}$. In both cases, the progenitor mass is close to the boundary mass between $\mathrm{BH}$ and NS formation (Nomoto et al. 2013). We emphasize that hydrodynamical modeling of the LC can provide better constraints on the highly uncertain progenitor masses of magnetars than the analytic prescription.

Our treatment of the SN evolution illustrates the importance of the dynamical effects on the ejecta, especially in cases of powerful magnetars. The homologous expansion, usually assumed in SN studies, can be broken because of the additional energy source. This could have an important effect on the line formation and the photospheric velocity evolution. For ASASSN-15lh, we found a total energy release by the magnetar of $E \sim 3 \times 10^{52} \mathrm{erg}$, which is one order of magnitude larger than the initial explosion energy. For the more moderate SN 2011kl, we obtained $E \sim 1.6 \times 10^{51} \mathrm{erg}$. In general, the dynamical effects on the expansion of the ejecta become significant when the magnetar energy is comparable to the explosion energy.

We are grateful to Sergei Blinnikov and Gastón Folatelli for helpful conversations and to Jose Prieto for sending the data of ASASSN-15lh. This research is supported by the World
Premier International Research Center Initiative (WPI Initiative), MEXT, Japan and the Grant-in-Aid for Scientific Research of the JSPS (23224004, 26400222), Japan. M.O. would like to thank Kavli IPMU for the support for her visit to Kavli IPMU.

\section{REFERENCES}

Arnett, W. D., \& Bowers, R. L. 1977, ApJS, 33, 415

Bersten, M. C., Benvenuto, O., \& Hamuy, M. 2011, ApJ, 729, 61

Bowers, R. L., Gleeson, A. M., \& Daryl Pedigo, R. 1975, PhRvD, 12, 3043

Dessart, L., Hillier, D. J., Li, C., \& Woosley, S. 2012, MNRAS, 424, 2139

Dexter, J., \& Kasen, D. 2013, ApJ, 772, 30

Dong, S., Shappee, B. J., Prieto, J. L., et al. 2015, arXiv:1507.03010

Gal-Yam, A. 2012, Sci, 337, 927

Greiner, J., Mazzali, P. A., Kann, D. A., et al. 2015, Natur, 523, 189

Hachinger, S., Mazzali, P. A., Taubenberger, S., et al. 2012, MNRAS, 422, 70

Inserra, C., \& Smartt, S. J. 2014, ApJ, 796, 87

Inserra, C., Smartt, S. J., Jerkstrand, A., et al. 2013, ApJ, 770, 128

Kasen, D., \& Bildsten, L. 2010, ApJ, 717, 245

Lattimer, J. M. 2012, ARNPS, 62, 485

Leloudas, G., Schulze, S., Krühler, T., et al. 2015, MNRAS, 449, 917

Lunnan, R., Chornock, R., Berger, E., et al. 2014, ApJ, 787, 138

Metzger, B. D., Margalit, B., Kasen, D., \& Quataert, E. 2015, arXiv: 1508.02712

Milisavljevic, D., James, D. J., Marshall, J. L., et al. 2015, ATel, 8216, 1

Moriya, T., Tominaga, N., Tanaka, M., Maeda, K., \& Nomoto, K. 2010, ApJL, 717, L83

Neill, J. D., Sullivan, M., Gal-Yam, A., et al. 2011, ApJ, 727, 15

Nomoto, K., \& Hashimoto, M. 1988, PhR, 163, 13

Nomoto, K., Kobayashi, C., \& Tominaga, N. 2013, ARA\&A, 51, 457

Quimby, R., Höflich, P., \& Wheeler, J. C. 2007, ApJ, 666, 1083

Quimby, R. M., Kulkarni, S. R., Kasliwal, M. M., et al. 2011, Natur, 474, 487

Shapiro, S. L., \& Teukolsky, S. A. 1983, Black Holes White Dwarfs and Neutron Stars: The Physics of Compact Objects (New York: WileyInterscience)

Smith, N., Li, W., Foley, R. J., et al. 2007, ApJ, 666, 1116

Sorokina, E., Blinnikov, S., Nomoto, K., Quimby, R., \& Tolstov, A. 2015, arXiv: 1510.00834

Swartz, D. A., Wheeler, J. C., \& Harkness, R. P. 1991, ApJ, 374, 266

Tanaka, M., Tominaga, N., Nomoto, K., et al. 2009, ApJ, 692, 1131

Timokhin, A. N., \& Harding, A. K. 2015, ApJ, 810, 144

Vietri, M., \& Stella, L. 1999, ApJL, 527, L43

Woosley, S. E. 2010, ApJL, 719, L204 